\title{
ARTICLE Opiate-associated contextual memory formation and retrieval are differentially modulated by dopamine D1 and D2 signaling in hippocampal-prefrontal connectivity
}

\author{
Yunpeng Wang ${ }^{1}$, Hongying Zhang ${ }^{2}$, Jingjing Cui ${ }^{1}$, Jing Zhang ${ }^{1}$, Fangyuan Yin ${ }^{1}$, Hao Guo ${ }^{1}$, Jianghua Lai ${ }^{1}$ and Bo Xing ${ }^{3,4}$
}

\begin{abstract}
Contextual memory driven by abused drugs such as opiates has a central role in maintenance and relapse of drug-taking behaviors. Although dopamine (DA) signaling favors memory storage and retrieval via regulation of hippocampal-prefrontal connectivity, its role in modulating opiate-associated contextual memory is largely unknown. Here, we report roles of DA signaling within the hippocampal-prefrontal circuit for opiate-related memories. Combining-conditioned place preference (CPP) with molecular analyses, we investigated the DA D1 receptor (D1R) and extracellular signal-regulated kinase (ERK)-CAMP-response element binding protein (CREB) signaling, as well as DA D2 receptor (D2R) and protein kinase B (PKB or Akt)/glycogen synthase kinase 3 (GSK3) signaling in the ventral hippocampus (VHip) and medial prefrontal cortex (mPFC) during the formation of opiate-related associative memories. Morphine-CPP acquisition increased the activity of the D1R-ERK-CREB pathway in both the vHip and mPFC. MorphineCPP reinstatement was associated with the D2R-mediated hyperactive GSK3 via Akt inhibition in the vHip and PFC. Furthermore, integrated D1R-ERK-CREB and D2R-Akt-GSK3 pathways in the vHip-mPFC circuit are required for the acquisition and retrieval of the morphine contextual memory, respectively. Moreover, blockage of D1R or D2R signaling could alleviate normal Hip-dependent spatial memory. These results suggest that D1R and D2R signaling are differentially involved in the acquisition and retrieval of morphine contextual memory, and DA signaling in the vHip-mPFC connection contributes to morphine-associated and normal memory, largely depending on opiate exposure states.
\end{abstract}

Neuropsychopharmacology (2019) 44:334-343; https://doi.org/10.1038/s41386-018-0068-y

\section{INTRODUCTION}

Learned associations between opiate use and environmental cues have a critical role in the maintenance and relapse of drug-taking after periods of abstinence [1]. The neurobiological substrate underlying these changes is linked to dopamine (DA) dysfunction originating from the ventral tegmental area (VTA) and the neuronal plasticity in the hippocampus (Hip) to prefrontal cortex (PFC) pathway, which carries contextual information about drug use. Both the Hip and PFC receive DA innervation from the VTA [2, 3] through multiple receptors, among which D1 receptor (D1R) and D2 receptor (D2R) are most abundant. Recent studies have revealed that noradrenergic fibers of the locus coeruleus also sends dopaminergic projections to the Hip, which facilitate hippocampus-dependent memory [4]. Disruption of Hip-PFC synchrony is associated with the cognitive deficits that occur in opiate addiction [5]. In particular, activation of D1R but not the $\mathrm{D} 2 \mathrm{R}$ is required for $\mathrm{N}$-methyl-D-aspartate (NMDA) receptordependent long-term potentiation (LTP) in Hip-PFC connections, which may underlie the transfer and storage of the contextual information [6]. However, once the memory is consolidated, D2R activation is required for further processing of information to guide future behaviors [7]. In this regard, dopamine D1 and D2 receptor signaling might exert distinct effects on opiate-related memory. In the Hip, both $\mathrm{D} 1 \mathrm{R}$ and $\mathrm{D} 2 \mathrm{R}$ are required for the formation of morphine-conditioned place preference (CPP) [8], whereas the role of D2R on reinstatement of morphine-CPP seems more significant than that of the D1R [9]. Nevertheless, the molecular mechanisms by which DA tone supports, opiateassociated contextual memories in the Hip-PFC circuit remain largely unknown.

It is well documented that the D1R couple to Gs G-proteins and activate adenylyl cyclases (AC) and protein kinase A (PKA), whereas the D2R couple to $\mathrm{Gi}$ or G0 G-proteins and inhibit AC and PKA [10, 11]. Activated D2 receptors also stimulate the inactivation of protein kinase $B$ (PKB or $A k t)$ by protein phosphatase $2 \mathrm{~A}(\mathrm{PP} 2 \mathrm{~A})$, leading to glycogen synthase kinase 3 (GSK3) hyperactivity [12]. Previous studies have shown that activation of D1R and PKA activate ERK through the DA- and CAMP-regulated phosphoprotein $32 \mathrm{kDa}$ signaling cascade [13]. Activated ERK translocates to the cell nucleus to regulate gene expression, including expression of those genes encoding the CAMP-response element binding protein (CREB) [14], which has an important role in the formation of normal learning and memory as well as drug-related memories [15]. Recent evidence has suggested that D2R activation mediates phosphorylation of Akt-GSK3 $\beta$ signaling, which contributes to the drug-conditioned rewards but not normal contextual learning [16]. Thus, it is not unexpected that D1R-ERK-CREB and D2R-Akt-GSK3 $\beta$ pathways in

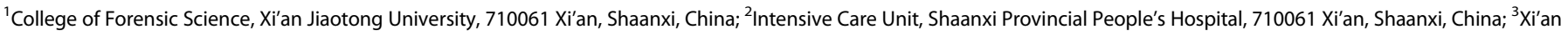
Mental Health Center, 710061 Xi'an, Shaanxi, China and ${ }^{4}$ Department of Neurobiology and Anatomy, Drexel University College of Medicine, Philadelphia, PA 19129, USA Correspondence: Bo Xing (bx32@drexel.edu)

Received: 1 January 2018 Revised: 1 April 2018 Accepted: 4 April 2018 Published online: 17 April 2018 
the Hip-PFC circuit have a distinctive role in opiate-associated contextual memory.

Here, we performed behavioral, electrophysiological and molecular experiments combined with pharmacological manipulations to determine how DA D1R and D2R signaling in the Hip-PFC circuit regulate the formation of opiate reward memories in opiate-naive and opiate-experienced states.

\section{MATERIALS AND METHODS}

The experimental procedures used in the present study are extensively described in Supplementary Materials and Methods. A similar procedure as described before [17] was used to establish CPP to morphine in mice. After CPP expression, extinction or reinstatement, animals were killed. Some brains from these mice were used for western blot analysis. For others, 300- $\mu \mathrm{m}$ coronal sections containing prefrontal cortex in which vHip-mPFC fibers are preserved were prepared as previously described [18-20]. After at least $10 \mathrm{~min}$ of stable baseline recording of a-amino-3-hydroxy-5-methyl-4-isoxazolepropionic acid receptor (AMPAR)-mediated excitatory postsynaptic current (EPSCs) from layer five pyramidal neurons, activity-dependent LTP was induced by three trains of $100 \mathrm{~Hz}$ stimulation of $1 \mathrm{~s}$ duration separated by $20 \mathrm{~s}$ of high-frequency stimulation (HFS) at baseline stimulus intensity. In addition, Morris water maze was used to detect the spatial memory ability, open-field test was used to examine the locomotion, and sucrose preference test was used to assess the degree of anhedonia after pharmacological manipulation or short hairpin RNA (shRNA)-mediated knockdown of DA D1R or D2R signaling.

\section{RESULTS}

Activation of D1R in the vHip and $\mathrm{mPFC}$ is necessary for morphineCPP expression

Mice were conditioned in an unbiased morphine-CPP paradigm (Fig. 1a), and LTP were recorded immediately after CPP testing. After four sessions of CPP training, the morphine-paired mice had a significant higher CPP score compared with all other groups (Fig. $1 \mathrm{~b}, n=12$ per group; $F_{\text {treatment } x \text { time }(2,66)}=16.57, p<0.0001$; post hoc, $p<0.0001)$, indicating that those mice preferred to spend more time in the morphine-paired chamber. We next determined whether the contextual-related morphine administration contributed to synaptic plasticity in the vHip-mPFC connection ex vivo. Specifically, we assessed the impact of hippocampal fiber bundle HFS stimulation on EPSC $A$ AMPAR responses from L5 prefrontal neurons. We found that HFS of the vHip fiber bundle resulted in LTP from saline controls, a form of plasticity that is absent in morphine-CPP mice (Fig. 1c-e). It is possible that changes in LTP may be directly caused by the repeated morphine administration itself instead of an effect of a morphine-paired distinct context. Therefore, an unpaired control morphine-CPP group was included that received morphine in both conditioning chambers. No change in CPP score was found in the morphineunpaired mice when compared with the saline group (Fig. 1b). Furthermore, normal LTP were induced in the morphine-unpaired mice (Fig. 1d, e). These results suggest that the deficits in activitydependent LTP of morphine-CPP mice cannot be ascribed to morphine exposure.

We next investigated potential changes in DA D1-regulated ERK-CREB signaling, as well as D2-regulated Akt-GSK3 $\beta$ signaling in the vHip and mPFC during the formation of opiate contextual memory. Representative western blots are presented in Fig. If. The analysis revealed that pERK 1 and $p$-CREB were both increased in vHip (pERK1: $F_{(2,15)}=13.39, p<0.0001 ; p$-ERK2: $F_{(2,15)}=1.35, p$ $=0.28 ;$ p-CREB: $\left.F_{(2,15)}=50.26, p<0.0001\right)$ and mPFC (pERK1: $F_{(2,}$ ${ }_{15)}=4.64, p<0.05 ; \mathrm{p}$-ERK2: $F_{(2,15)}=0.68, p=0.55$; $\mathrm{p}$-CREB: $F_{(2,15)}$ $=10.5, p<0.01)$ in the morphine-paired group. The morphine- unpaired mice showed similar pERK $1 / 2$ and $\mathrm{p}$-CREB expression in the vHip and mPFC to saline mice. In contrast, no difference was found in phosphorylated Akt (Thr308 in Fig. If and Ser473 in Supplementary Figure S1A) and GSK3 $\beta$ levels in the vHip and mPFC in morphine-paired mice following CPP expression. We also tested and found that the pERK1/pAkt ${ }^{\text {Thr308 }}$ signaling is directly linked to D1R/D2R activity, respectively (Supplementary Figure S2). Thus, we hypothesized that upregulation of ERK-CREB signaling in vHip and MPFC may be linked to the activation of $\mathrm{D} 1 \mathrm{R}$ transmission in those regions during the formation of morphine contextual memory. Accordingly, we next examined the effects of D1R knockdown directly on the effect of vHip and mPFC on CPP expression. Mice were intra-vHip and $\mathrm{mPFC}$ injected with AAV-expressed shDrd1 or Scr shRNA 2 weeks prior to the CPP experiment ( $n=8$ per group). Interestingly, the knockdown of D1R in both vHip and mPFC blocked morphine-CPP expression in comparison with the robust CPP in the Scr controls (Fig. 1g, h). No significant difference in the total distance traveled or sucrose preference was observed between the shDrd1 and Scr-injected mice, indicating intact locomotor activity and rewarding responses in those mice (Supplementary Figure S1B, C).

We next examined whether the observed changes in LTP and D1-ERK-CREB signaling following CPP expression remained after morphine withdrawal. A separate group of mice $(n=6$ for saline group and $n=8$ for morphine group) were conditioned and tested for morphine-CPP as described above. After CPP expression, the mice were kept in their home cages for 7 days and thus not exposed to the drug-related context anymore (Fig. 2a). After 7 days of home-cage withdrawal, the morphine group still showed strong preference for the morphine-paired chamber (Fig. 2b; day 16 , morphine vs. saline, $t_{12}=3.642, p<0.01$ ). Another cohort of mice ( $n=8$ per group) were sequentially underwent CPP training and 7 days of home-cage withdrawal and was killed for LTP test on day 16. The HFS-triggered LTP in morphine-paired mice was indistinguishable from that in saline mice 7 days after morphine home-cage withdrawal (Fig. 2c, d). No significant difference was found in pERK1/2 and p-CREB expression levels in both vHip and mPFC between the morphine-paired mice and the saline controls (Fig. 2e). These results showed that changes in LTP and D1R signaling in the vHip-mPFC circuit were extinguished when mice were no longer exposed to the previously morphine-paired context. Therefore, it can be concluded that the decrease in LTP only accompany the acquisition of morphine-CPP but not necessarily its expression.

We also examined the effect of viral-mediated D2R downregulation in vHip and $\mathrm{mPFC}$ on morphine-CPP acquisition. D2R knockdown in vHip or mPFC had no significant effect on morphine-CPP acquisition (Supplementary Figure S3).

D2R activation in the vHip and mPFC is necessary for morphineCPP reinstatement

To investigate the roles of D1R and D2R in the vHip and mPFC during the retrieval of opiate contextual memory, we next examined the electrophysiological and molecular changes in mice following the morphine-CPP extinction and reinstatement. Mice were conditioned and tested with morphine-CPP acquisition and then extinction procedures (Fig. 3a). Mice in the two morphinepaired groups both showed significant preferences for the morphine-paired chamber, whereas the morphine-unpaired groups did not form a preference for a particular context (Fig. 3b; $n=12$ per group; $\left.F_{(3,44)}=11.5, p<0.0001\right)$. After four sessions of extinction training, the CPP scores in the morphine-paired groups became indistinguishable from those of the saline-paired controls, suggesting a successful extinction of morphine-CPP. Twenty-four hours after the extinction test, mice were challenged with a single dose of morphine ( $5 \mathrm{mg} / \mathrm{kg}$, i.p.) or saline and were tested for CPP restatement. The preference for the previous morphine-paired 
A
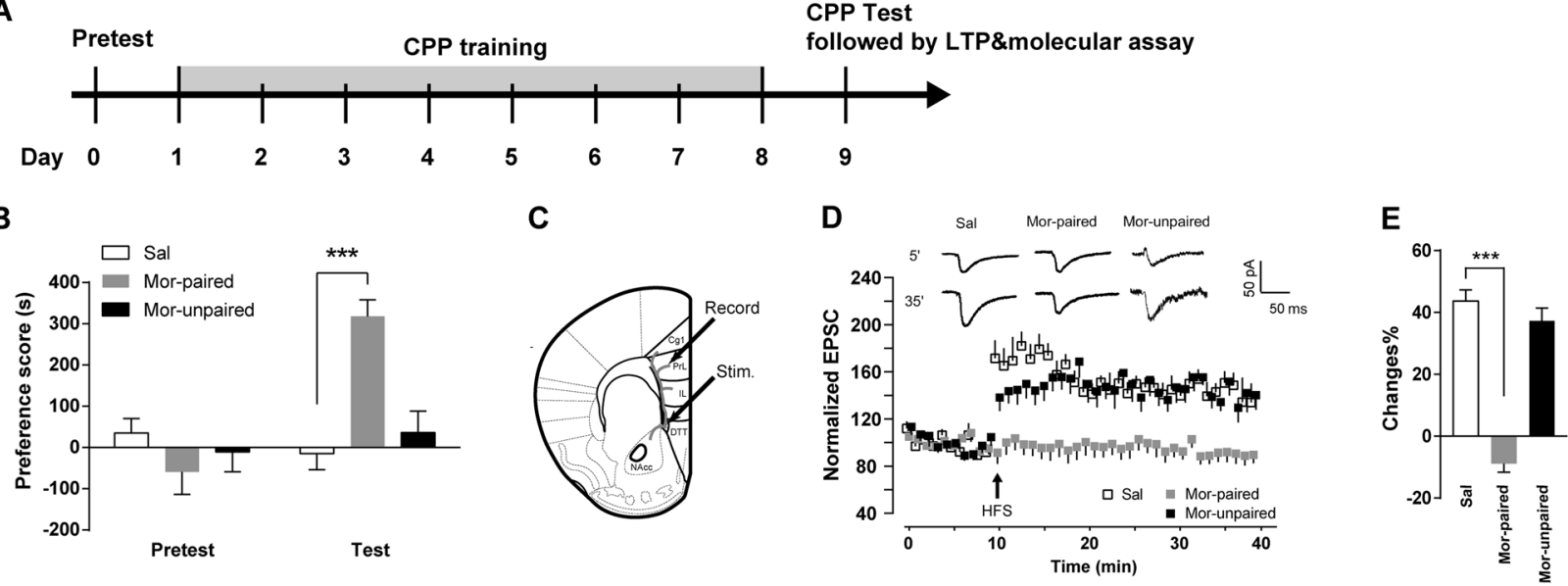

$\mathbf{F}$

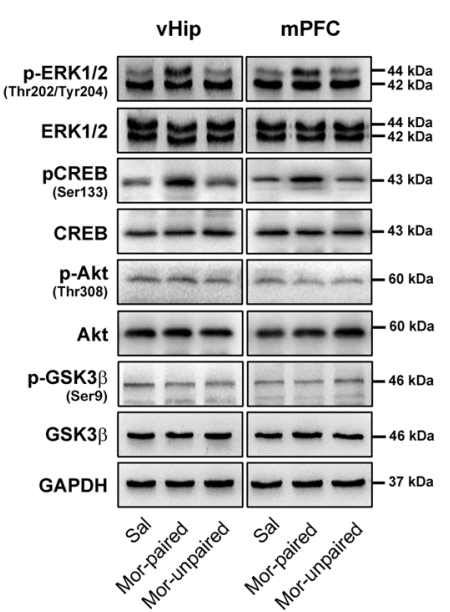

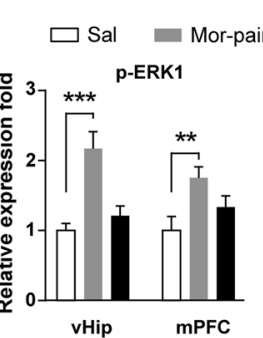

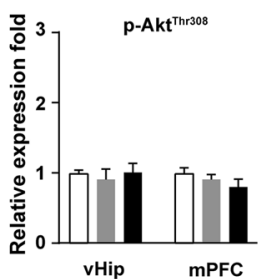

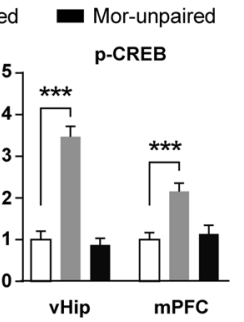

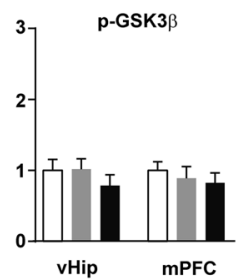

$\mathrm{G}$
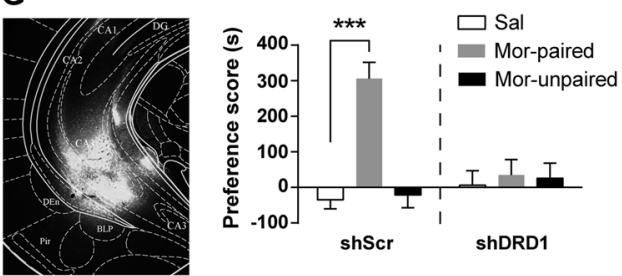

H
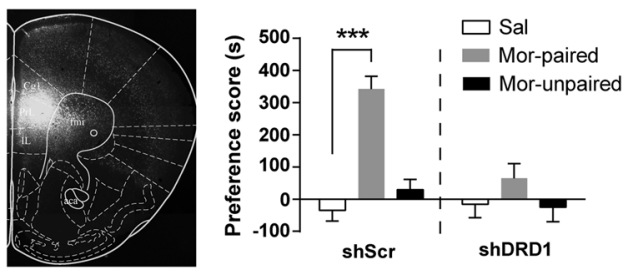

Fig. 1 Activation of D1R in the ventral hippocampus (vHip) and medial prefrontal cortex (mPFC) is necessary for morphine-conditioned place preference (CPP) expression. a Schematic experimental design for the training and testing of morphine-CPP. b Morphine (10 mg/kg, i.p.) induced CPP expression after four training sessions. $n=12$ per group. c Diagram illustrating the experimental design used to assess the synaptic plasticity in the vHip-mPFC connection ex vivo. d Summary graph showing no high-frequency stimulation (HFS)-induced LTP in the morphine-paired group. e There are significant deficits in LTP induction in the morphine-paired group but not in the saline or morphineunpaired group. $\mathbf{f}$ Representative western blot and phosphorylated ERK1/2 (pERK1/2), p-CREB, pAkt, pGSK3 $\beta$ immunoreactivity in the vHip and mPFC. g Left, representative microphotograph of shDRD1 expression in vHip. Right, effect of D1R knockdown in vHip on morphine-CPP expression, in comparison with the scramble shRNA (Scr) controls. $n=8$ per group. $\mathbf{h}$ Representative microphotograph of shDRD1 expression in mPFC (left) and effect of D1R knockdown in both mPFC on morphine-CPP expression (right). $n=8$ per group. Error bars show the standard error of the mean (SEM). ${ }^{* *} p<0.01,{ }^{* * *} p<0.0001$

chamber was reinstated significantly in the morphine-paired + morphine-challenged mice, whereas the morphine-paired + saline-challenged and morphine-unpaired + morphine-challenged mice did not show any place preference for either chamber $\left(F_{(3,44)}=14.27, p<0.0001\right)$.

We next assessed the effect of the hippocampal fiber bundle

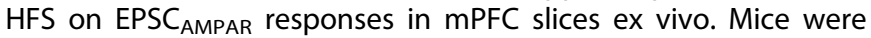
killed immediately following the CPP reinstatement test and brains were quickly sliced for LTP assay (slices were prepared about 60 min after final reinstatement test) or stored in $-70^{\circ} \mathrm{C}$ for further western blot assay. As is showed in Fig. $3 c$ and d, only neurons from morphine-CPP reinstatement mice did not exhibit LTP after HFS. These results indicated that the LTP was impaired in the vHip-mPFC circuit following the reinstatement of morphine-CPP.

We also examined changes in protein levels for DA D1- and D2regulated signaling cascades during the retrieval of contextual opiate memories. Representative western blots are presented in Fig. 3e. One-way ANOVA revealed a significant decrease of pAkt phosphorylation at the Thr308 site in both the vHip $\left(\mathrm{F}_{(3,28)}=\right.$ 4.179, $p<0.05)$ and $\operatorname{mPFC}\left(F_{(3,28)}=9.623, p<0.01\right)$ of the morphine-paired + morphine-challenged mice, whereas no difference was found in pAkt phosphorylation at the Ser473 site in any of the groups (Supplementary Figure S4A). Moreover, the morphine-paired + morphine-challenged mice showed similar decreases in pGSK3 $\beta$ levels in the vHip $\left(F_{(3,28)}=3.846, p<0.05\right)$ and $\operatorname{mPFC}\left(F_{(3,28)}=7.908, p<0.01\right)$ compared with that in other groups. No difference was found in p-ERK and p-CREB levels in the vHip and mPFC among the four groups. Together, these results indicated that the Akt-GSK3 $\beta$ signaling cascade but not the ERK-CREB signaling cascade was activated following the reinstatement of expressed morphine-CPP.

We next examined the effects of local antagonism of D2R in the vHip and mPFC on reinstatement of morphine-CPP. Mice were trained and tested with morphine-CPP acquisition and then subjected to extinction procedures ( $n=8$ per group). After the extinction test, stereotaxic surgery and cannulas implantation were performed, followed by a 5-day recovery period. On the CPP 
A
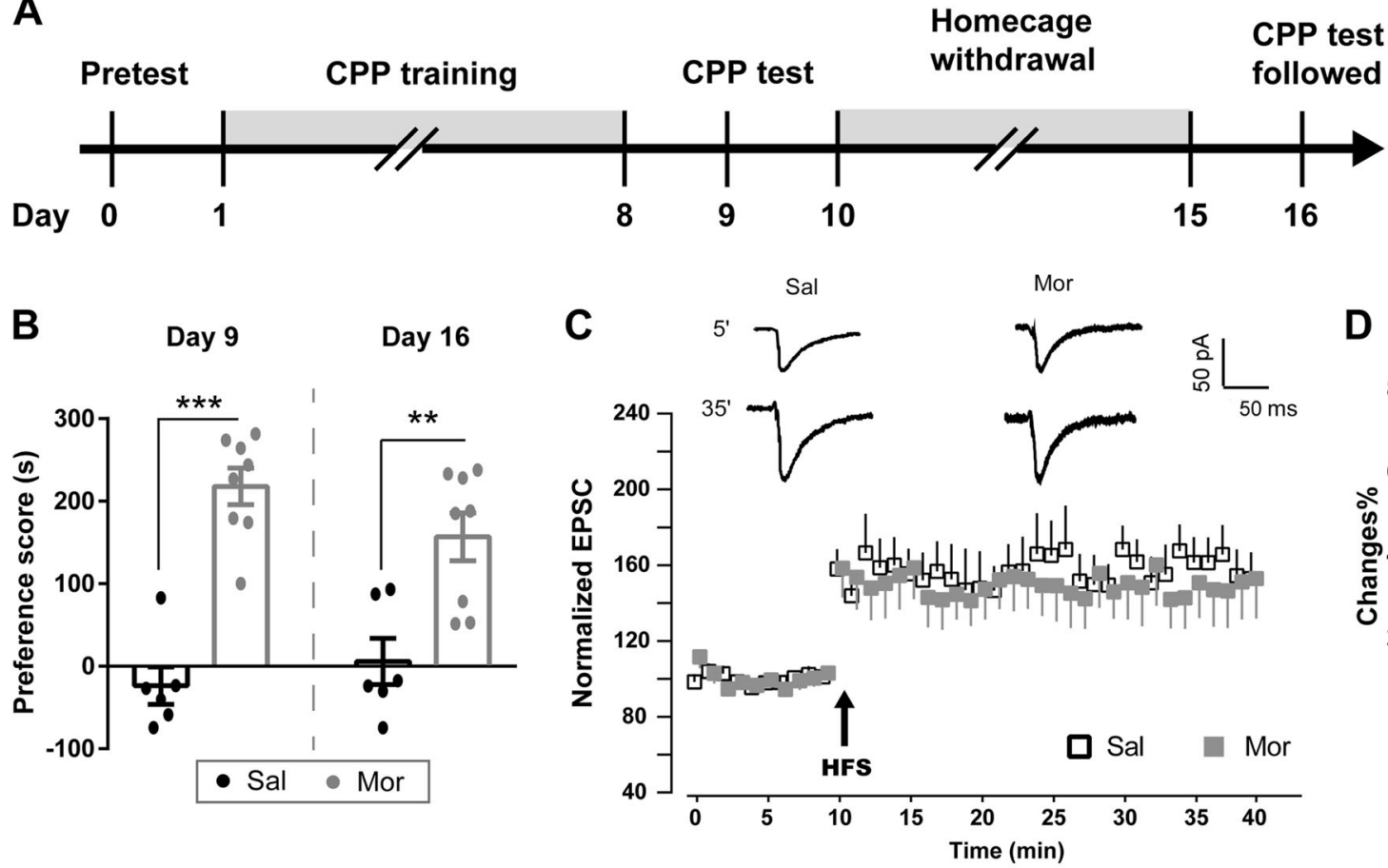

D

E
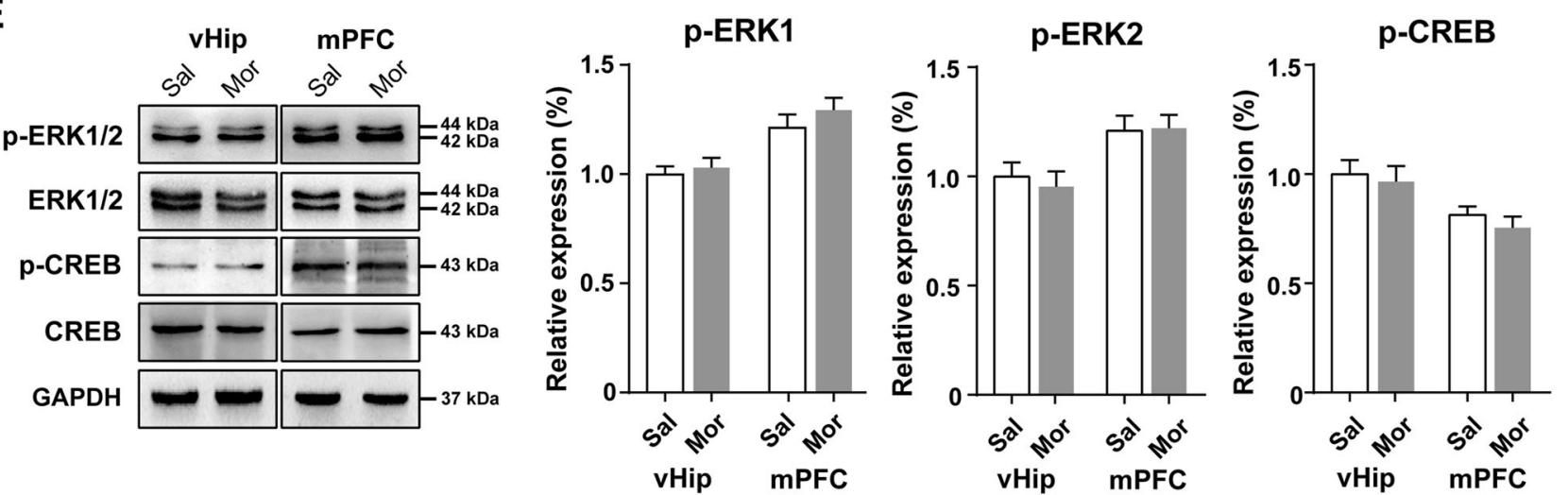

Fig. 2 Changes in LTP and D1-ERK-CREB signaling following CPP expression remain after morphine withdrawal. a Schematic experimental design for morphine-CPP and spontaneous withdrawal. $\mathbf{b}$ CPP scores on day 9 and $16 .{ }^{* *} p<0.01$, ${ }^{* *} p<0.0001$ compared between the salineand morphine-treated mice ( $n=8$ per group). c, d High-frequency-induced LTP is intact in both the saline and morphine-CPP groups. e Representative western blot showing $\mathrm{pERK} 1 / 2$ and $\mathrm{p}$-CREB immunoreactivity in the vHip and mPFC. Error bars show the standard error of the mean (SEM)

reinstatement test day, mice were microinjected with a selective D2R antagonist raclopride $(5 \mu \mathrm{g} / 0.5 \mu \mathrm{l} / \mathrm{side})$ or DMSO into the vHip or mPFC. Thirty minutes later, they were i.p. injected with a priming dose of morphine or saline. As showed in Fig. 3f, reinstatement of expressed morphine-CPP was fully blocked by infusion of raclopride into the vHip or MPFC in comparison with the significant CPP reinstatement in the morphine-challenged DMSO controls. No significant difference in the total distance traveled or sucrose preference was observed between the raclopride and DMSO injected mice, indicating intact locomotor activity and rewarding responses in those mice (Supplementary Figure S4B-D).

Integrated D1-ERK signaling in the vHip-mPFC circuit is required for the formation of opiate contextual memory

So far, our results show that D1R (but not D2R) signaling in the vHip and mPFC is necessary for the formation of the opiate contextual memory. We next investigated whether integrated D1-
ERK signaling in the vHip-mPFC circuit is necessary for the formation of opiate contextual memory. We tested this hypothesis with different combinations of intra-vHip or intra-mPFC MEK1 inhibitor (PD334581, $1 \mu \mathrm{g} / 0.5 \mu \mathrm{l} / \mathrm{side}$ ) in the same or different hemispheres before morphine-CPP conditioning (Fig. 4a). Twoway ANOVA revealed a significant interaction between the conditioning environment and treatment $\left(F_{(5,} 84\right)=2.437$, $p<0.05 ; n=8$ per group; Fig. 4b). Unilateral blockade of ERK activity in MPFC or vHip in one hemisphere (group II and III; in each group, half of the mice were injected with PD334581 in the left hemisphere and another half of mice in the right hemisphere) did not affect the expression of morphine-CPP. However, morphine-CPP was prevented when mice were injected with PD334581 into the unilateral vHip and contralateral mPFC (group IV). Interestingly, when compared with group V (mice were microinjected with saline in the same hemisphere), there was a significant decrease $(p<0.05)$ in morphine-CPP score in group VI (mice were microinjected with PD334581 in 
A

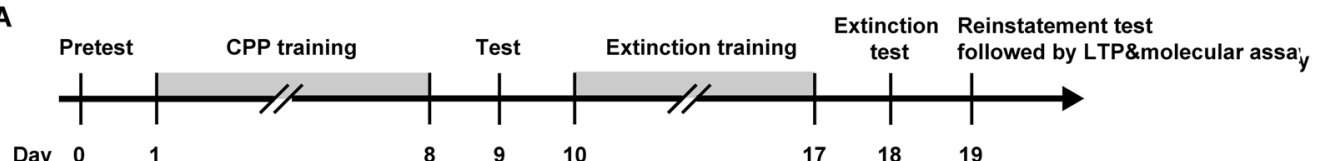

Day $0 \quad 1$

D

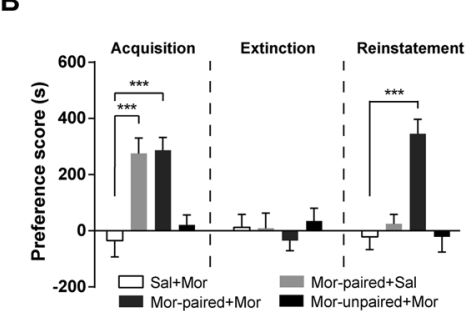

C
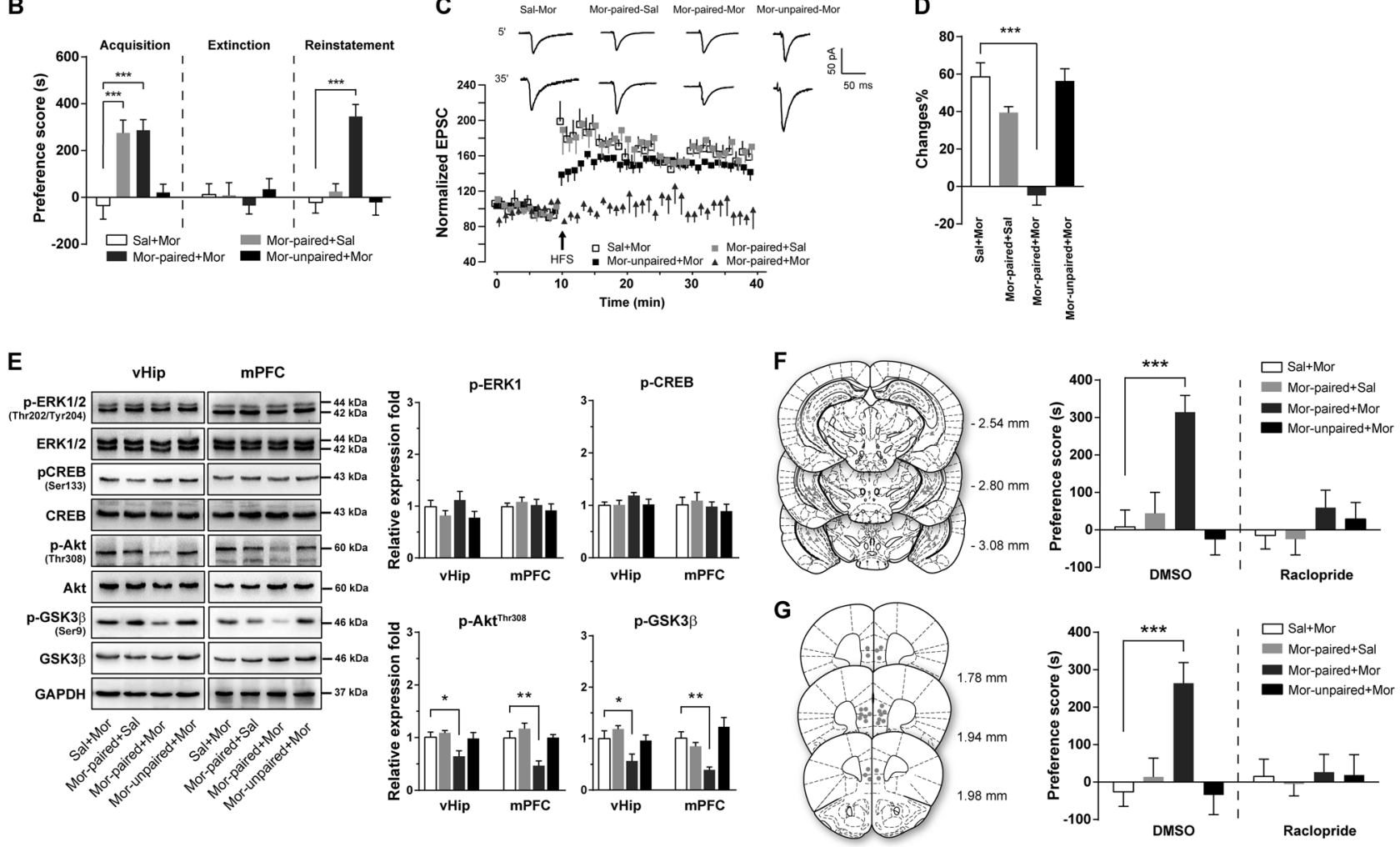

Fig. 3 Integrated D2-Akt signaling in the vHip-mPFC circuit is required for the retrieval of opiate contextual memory. a Schematic experimental design for the training and testing of morphine-CPP acquisition, extinction, and reinstatement. b Scores in morphine-CPP expression, extinction, and reinstatement tests. $n=12$ per group. $\mathbf{c}$, d Synaptic plasticity in the vHip-mPFC connection was assessed following a morphine-CPP reinstatement test. Only the morphine-paired with morphine challenge group shows obvious impairment in LTP induction. $\mathbf{e}$ Representative western blot and pERK1/2, p-CREB, pAkt, pGSK3 $\beta$ immunoreactivity in the vHip and mPFC. f On the reinstatement test day, mice were microinjected with raclopride $(5 \mu \mathrm{g} / 0.5 \mu \mathrm{l} / \mathrm{side}$ ) or DMSO into the vHip or $\mathrm{mPFC}$ ( $n=8$ per group). Thirty minutes later, they were i.p. injected with a priming dose of morphine $(5 \mathrm{mg} / \mathrm{kg})$ or saline. Left, schematic of bilateral intra-vHip guide cannula and microinjection tip placements. Right, effect of intra-vHip administration with raclopride on morphine-CPP expression, in comparison with the DMSO controls. $\mathbf{g}$ Left, schematic of bilateral intra-mPFC guide cannula and microinjection tip placements. Right, effect of intra-mPFC administration with raclopride on morphine-CPP reinstatement. Error bars show the standard error of the mean (SEM). ${ }^{*} p<0.05,{ }^{* *} p<0.01,{ }^{* * *} p<0.0001$

the vHip-mPFC circuit in the same hemisphere), although these mice had significant morphine-CPP compared with its saline counterparts.

We also examined the effect of ERK blockade in the vHip-mPFC circuit on a morphine-CPP reinstatement. Mice were trained and tested with morphine-CPP acquisition procedure and then a CPP extinction procedure. Then, PD334581 $(1 \mu \mathrm{g} / 0.5 \mu \mathrm{l} / \mathrm{side})$ was microinjected into the vHip or mPFC in different combinations in mice $30 \mathrm{~min}$ before they were challenged with a priming dose of morphine. No effect of PD334581 treatment was found on the morphine-CPP reinstatement $\left(F_{\mathrm{PD} 334581}(5,84)=1.889, p=0.1048\right.$; $n=8$ per group; Fig. $4 c$ ). All mice from different treatment groups demonstrated a significant reinstatement of preference for the previous morphine-paired environment $\left(F_{\text {reinstatement }(1,84)}=36.96\right.$, $p<0.0001)$.

Integrated D2-Akt signaling in the vHip-mPFC circuit is required for the retrieval of opiate contextual memory

As we have shown hyperactive D2R correlated with reinstatement, it is reasonable to investigate whether integrated D2R-Akt signaling in the vHip-mPFC circuit is necessary for the retrieval of opiate contextual memory. Mice were treated with different combinations of intra-vHip or intra-mPFC Akt activation (SC79, 6 $\mu \mathrm{g} / 0.5 \mu \mathrm{l} / \mathrm{side}$ ) in the same or different hemispheres before the morphine-CPP conditioning (Fig. 5a). There was no observed effect of the treatments (Fig. 5 b), although we did observe an effect of morphine conditioning $\left(F_{(1,84)}=64.82, p<0.0001 ; n=8\right.$ per group). We then examined the effect of Akt activation in the vHip-mPFC circuit on morphine-CPP reinstatement. Mice were trained and tested with a morphine-CPP acquisition procedure and then a CPP extinction procedure. Then, SC79 $(6 \mu \mathrm{g} / 0.5 \mu \mathrm{l} / \mathrm{side})$ was microinjected into the vHip or MPFC in different combinations $30 \mathrm{~min}$ before a priming dose of morphine. We found that pharmacological activation of Akt in the vHip-mPFC circuit prevented morphine-CPP reinstatement (Fig. 5c). Unilateral activation of Akt activity in vHip or mPFC in different hemispheres (group II and III) did not affect the formation of morphine-CPP. However, morphine-CPP reinstatement was fully prevented when mice were unilaterally injected with SC79 in the vHip paired with contralateral SC79 in MPFC (group IV) because these mice showed no preference for the morphine-paired chamber after the challenge. 
A
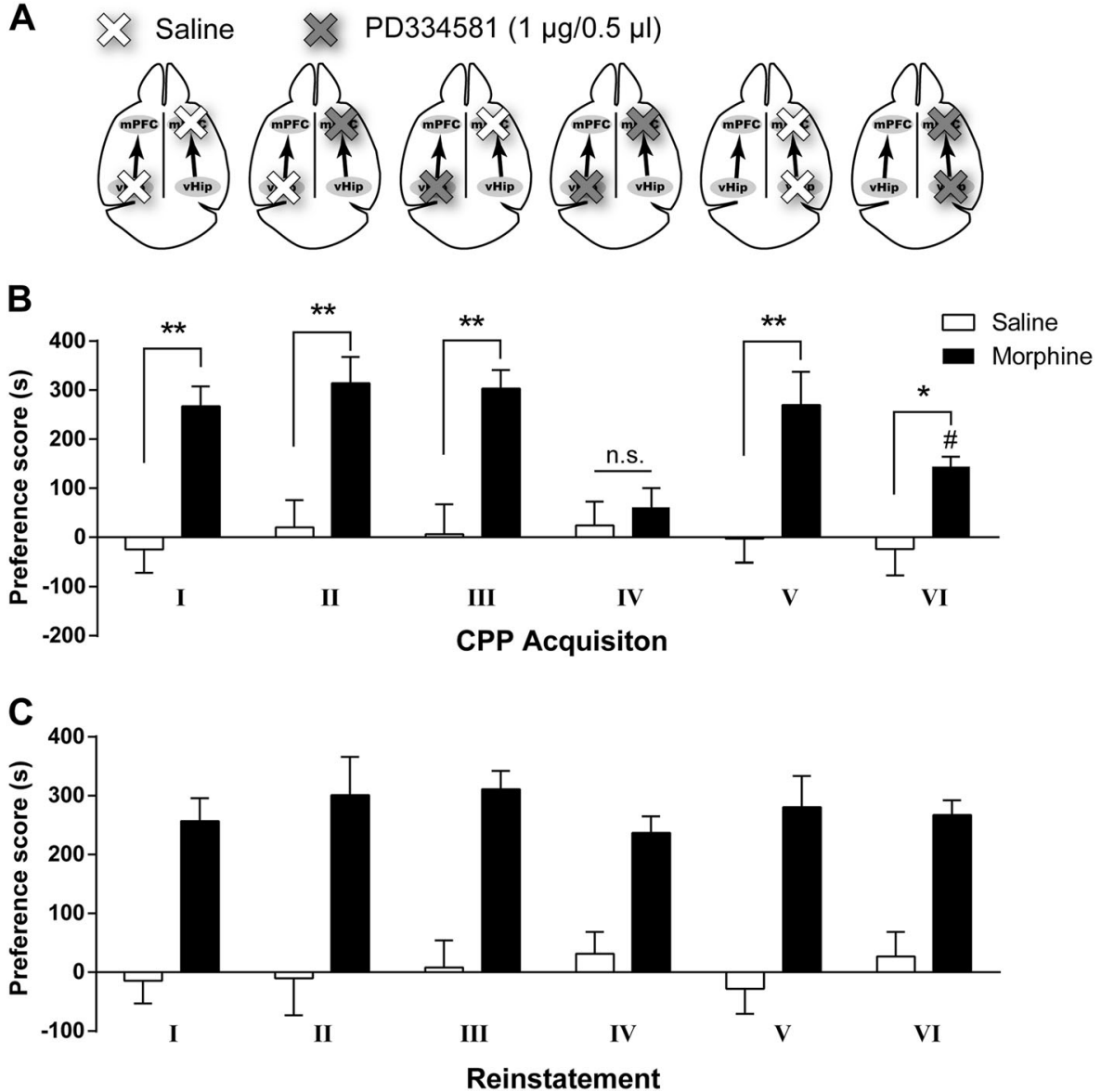

Fig. 4 Integrated D1-ERK signaling in the vHip-mPFC circuit is required for the formation of opiate contextual memory. a Schematic representation of an experimental protocol for blocking ERK signaling during acquisition/reinstatement of morphine-CPP. $\mathbf{b}$ Unilateral intravHip combined with contralateral intra-mPFC microinfusion of PD334581 (1 $\mu \mathrm{g} / 0.5 \mu \mathrm{l} / \mathrm{side}$ ) blocked the acquisition of a morphine (10 mg/kg) CPP. $n=8$ per group. c ERK blockade by PD334581 in the vHip-mPFC circuit did not affect morphine-CPP reinstatement. $n=8$ per group. ${ }^{*} p<$ $0.05,{ }^{* *} p<0.01 ;{ }^{\#} p<0.05$ between the morphine-treated groups for group $\mathrm{V}$ and VI. Error bars represent the SEM

A previous study has shown that D2R knockout induces both increase of pAkt at Thr308 and decrease of pAkt at Ser473 under basal conditions in striatum [21], suggesting a complicated Akt regulation by D2 stimulation. Our data have shown that reinstatement of morphine-CPP lead to a decrease of $\mathrm{pAkt}^{\text {Thr308 }}$ (Fig. 3e) with unaffected pAkt ${ }^{\text {Ser473 }}$ (Supplementary Figure S4A). Furthermore, unilateral intra-vHip combined with contralateral intra-mPFC microinfusion of the Akt activator SC79 (Fig. 5c) but not the Akt antagonist 10-DEBC $(0.5 \mu \mathrm{g} / 0.5 \mu \mathrm{l} / \mathrm{side}$, Supplementary Figure S5) blocked the reinstatement of morphine-CPP, suggesting the D2-pAkt ${ }^{\text {Thr308 }}$-pGSK3 ${ }^{\text {Ser9 }}$ signaling pathway directly involved in the reinstatement process.

The morphine-induced impairment of spatial reference memory in mice is restored by blocking D1 and D2 signaling in the vHip-mPFC circuit depending on the opiate exposure state To address whether normal learning and memory ability interfere with formation/retrieval of opiate contextual memory, mice spatial reference memory was examined by a Morris water maze task during morphine-CPP expression and reinstatement $(n=8$ per group). The timeline of the experiment is shown in Fig. 6a. The water maze training, which was performed $5 \mathrm{~h}$ following CPP training, was conducted in parallel with the morphine-CPP conditioning or extinction training procedures, respectively. Two-way repeated measures ANOVA indicated a significant main effect of treatment $\left(F_{(3,224)}=18.96, p<0.0001\right)$ and time $\left(F_{(7,224)}\right.$ $=25.32, p<0.0001)$ on escape latency, with no treatment $x$ time interaction for either measure (Fig. 6b). In addition, no significant difference was observed among all of the groups in average swimming speed, indicating that the overall locomotor activity was not affected throughout the training sessions (see Supplementary Figure S6). Spatial memory retention of the platform location was assessed in the probe test performed on the day when mice were tested for morphine-CPP expression and reinstatement. The representative swimming tracks of the mice are shown in Fig. $6 \mathrm{c}$. The time spent in the target quadrant $\left(F_{(3,28)}\right.$ $=6.188, p<0.01$; Fig. $6 \mathrm{~d})$ and in platform crossing $\left(F_{(3,28)}=6.947\right.$, $p<0.01$; Fig. 6e) were significantly affected by the treatments. Mice that expressed morphine-CPP spent significantly less time in the target quadrant (post hoc, $p<0.01$ ) and had fewer platform crossings $(p<0.05)$ than mice in the saline group. Moreover, morphine-challenged CPP-expressed mice also showed decreased time in the target quadrant $(p<0.05)$ and had fewer platform crossings $(p<0.05)$ than the saline-challenged mice. These results suggest that normal spatial memory was impaired during both morphine-CPP formation and reinstatement.

So far, our data have shown that opiate contextual memory formation and retrieval requires hyperactive $D 1 R$ and $D 2 R$ signaling in the vHip-mPFC circuit, respectively, and spatial reference memory was impaired during these processes. We propose that the impairments in spatial memory may be alleviated by intra-vHip-mPFC D1R or D2R signaling blockage during morphine-CPP formation and reinstatement, respectively. To test this hypothesis, mice were treated with different 
A

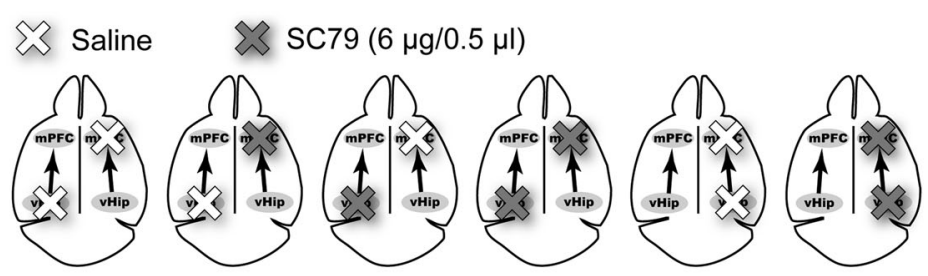

B
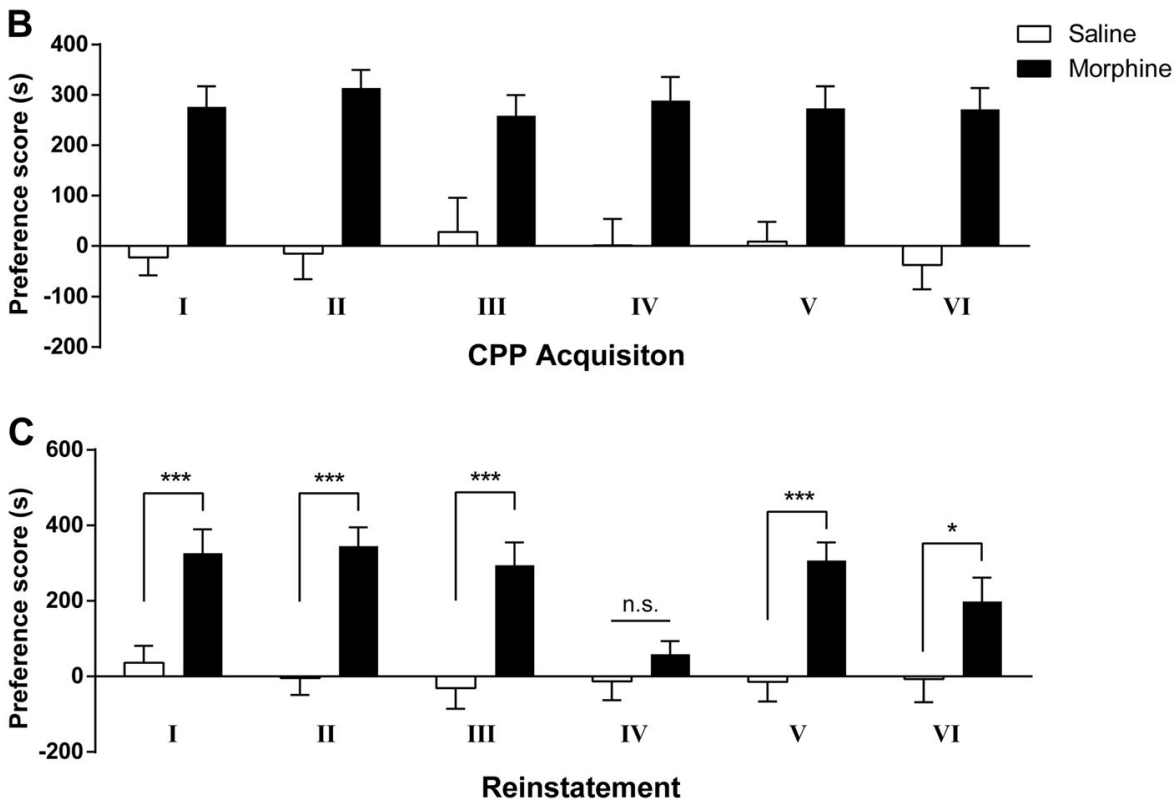

Fig. 5 Integrated D2-Akt signaling in the vHip-mPFC circuit is required for the retrieval of opiate contextual memory. a Schematic representation of the experimental protocol for activating Akt signaling during acquisition/reinstatement of morphine-CPP. $\mathbf{b}$ Activation of Akt signaling by SC79 $(6 \mu \mathrm{g} / 0.5 \mu \mathrm{l} / \mathrm{side})$ in the vHip-mPFC circuit did not affect morphine-CPP acquisition. $n=8$ per group. c Unilateral intra-vHip combined with contralateral intra-mPFC microinfusion of SC79 blocked the reinstatement of morphine-CPP. $n=8$ per group. ${ }^{*} p<0.05$, ${ }^{* * *} p<$ 0.0001. Error bars represent the SEM

combinations of selective D1R antagonist SCH23390 $(1 \mu \mathrm{g} / 0.5 \mu \mathrm{l} /$ side) or D2R antagonist raclopride $(5 \mu \mathrm{g} / 0.5 \mu \mathrm{l} / \mathrm{side})$ in the vHip or mPFC in the same or different hemispheres before morphine-CPP conditioning ( $n=8$ per group). The water maze training was performed $5 \mathrm{~h}$ following the CPP training. Pretreatment with $\mathrm{SCH} 23390$ but not raclopride during CPP conditioning significantly attenuated the CPP acquisition (Supplementary Figure S7). As shown in Fig. 6f, during morphine-CPP formation, the mice in group III (unilateral intra-vHip D1R blockade paired with contralateral mPFC D1R blockade) spent a similar amount of time in the target quadrant as mice in group I (saline controls), suggesting that the morphine-CPP formation-induced water maze deficit was fully prevented. Simultaneously blocking D1R signaling in the vHip-mPFC connection in the same hemisphere (group IV) did not prevent the CPP-induced water maze deficit (vs. group I, $p<0.05$ ). However, a post hoc test revealed a significant difference $(p<0.05)$ between group IV and II (morphine-CPP mice microinjected with saline in the vHip and mPFC), suggesting that the spatial memory deficit was partially ameliorated. In contrast, blockade of intra-vHip-mPFC D2R signaling (group V and VI) had no effect on water maze performances accompanying morphineCPP formation.

We then assessed the effect of intra-vHip-mPFC D1R and D2R signaling blockage on CPP reinstatement-induced spatial memory deficit. CPP-expressed mice were trained with a CPP extinction procedure. The Morris water maze training was performed $5 \mathrm{~h}$ following the CPP extinction training. On the reinstatement test day, mice were treated with different combinations of $\mathrm{SCH} 23390$ ( $1 \mu \mathrm{g} / 0.5 \mu \mathrm{l} / \mathrm{side})$ or raclopride $(5 \mu \mathrm{g} / 0.5 \mu \mathrm{l} / \mathrm{side})$ in the vHip or mPFC before the i.p. morphine challenge ( $n=8$ per group). A water maze probe test was performed $5 \mathrm{~h}$ following the CPP reinstatement test. As shown in Fig. $6 \mathrm{~g}$, the mice in group $\mathrm{V}$ (unilateral intra-vHip D2R blockade paired with contralateral mPFC D2R blockade) spent a similar amount of time in target quadrant as the saline controls (group I), suggesting that the morphine-CPP reinstatement-induced spatial memory deficit was reversed. There was a significant difference $(p<0.01)$ between group VI (morphine-challenged morphine-CPP-expressed mice microinjected with raclopride in the vHip and $\mathrm{mPFC}$ in the same hemisphere) and group II (morphine-challenged morphine-CPP-expressed mice microinjected with saline in the vHip and $\mathrm{mPFC}$ ), suggesting an improvement in spatial memory. As expected, blockade of intravHip-mPFC D1R signaling showed no obvious effect on CPP reinstatement-induced spatial memory deficit.

\section{DISCUSSION}

It is generally accepted that vHip-mPFC interactions are critically involved in regulation of mnemonic processes. However, it is difficult to explain continued opiate use results of enhanced drugtriggered contextual memories that are concurrent with deficits in hippocampal LTP and hippocampus-dependent learning and memory. Laboratory rodents successfully learn CPP to morphine, suggesting that normal drug-context learning occurs through the reinforcing effects of the drug. One possibility is that opiaterelated cues are flagged as salient and grab the drug users' attention and impair the context-dependent memory in a natural environment, and the potential substrate underlying the process is the vHip-mPFC connection with DA signaling as the initial cause of adaptive behavior induced by opiates. 
A
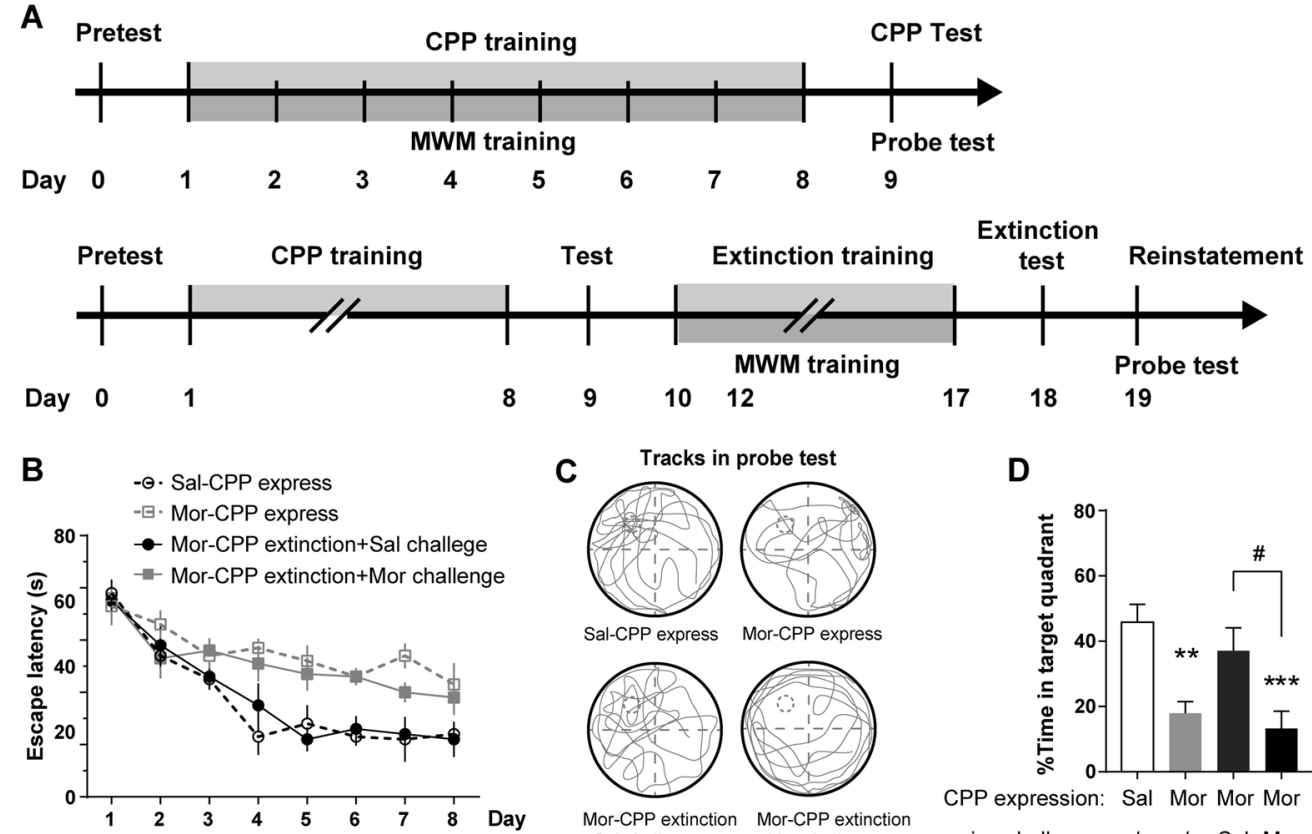

C Tracks in probe test

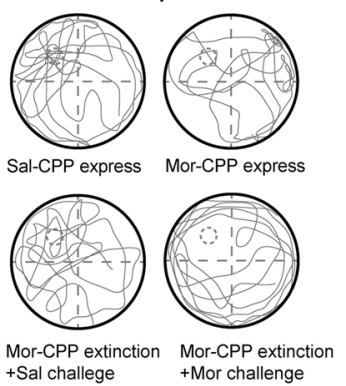

D

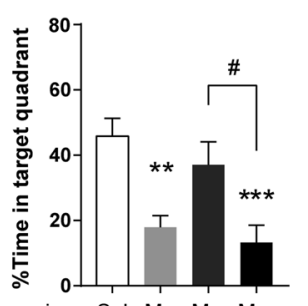

CPP expression: Sal Mor Mor Mor

i.p. challenge: / / Sal Mor
E

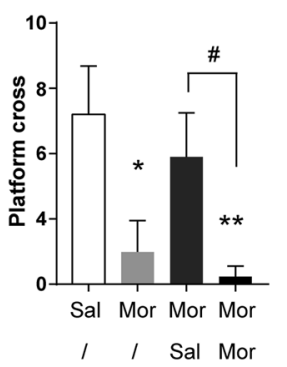

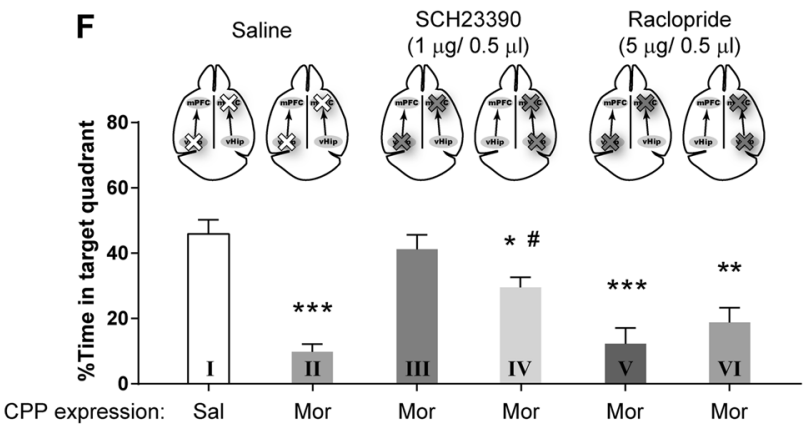

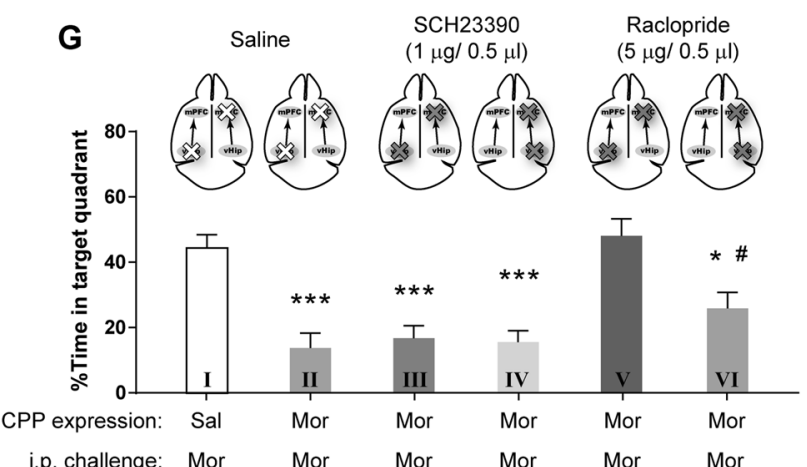

Fig. 6 The morphine-induced impairment of spatial reference memory in mice is restored by blocking D1 and D2 signaling in the vHip-mPFC circuit depending on the opiate exposure state. a Schematic representation of the experimental protocol for pairing the morphine-CPP acquisition and extinction (9:00 a.m.) with the Morris water maze task (2:00 p.m.). b Learning curves for the mean escape latency during the water maze acquisition. $n=8$ per group. $\mathbf{c}$ Representative swimming paths of mice in the Morris water maze probe test. $\mathbf{d}$ Percentage of time spent in the target quadrant and e number of platform crossings in the Morris water maze probe test. ${ }^{*} p<0.05,{ }^{* *} p<0.01,{ }^{* * *} p<0.0001$ compared with the saline group. $\mathbf{f}$ Effect of D1- or D2-signaling blockade in the vHip-mPFC circuit on water maze performance in the morphine-CPP-expressed mice. $n=8$ per group. ${ }^{*} p<0.05,{ }^{*} p<0.01,{ }^{* * *} p<0.0001$ compared with the saline group (group I). ${ }^{\#} p<0.05$ compared with group II (morphine-CPP mice microinjected with saline in the vHip and mPFC). $\mathbf{g}$ Effect of D1- or D2-signaling blockade in the vHip-mPFC circuit on water maze performance after reinstatement of morphine-CPP in mice. $n=8$ per group. ${ }^{*} p<0.05$, ${ }^{* * *} p<0.0001$ compared with the saline group (group I). $" p<0.05$ compared with group II (morphine-challenged morphine-CPP-expressed mice microinjected with saline in the vHip and mPFC)

Our study has revealed that in opiate-naive mice, D1R-mediated signaling within the vHip-mPFC connection is required for morphine reward memory, which switches to a D2R-dependent signaling substrate following chronic morphine exposure and withdrawal. Moreover, the vHip to mPFC LTP and spatial reference memory deficits are observed, regardless of exposure states. Interestingly, blockage of D1R for drug-naive mice or of D2R for drug-experienced animals in vHip and mPFC of one hemisphere (either left or right hemisphere) can reverse the impairments in hippocampal-dependent spatial memory.

It is well established that D1R activation in the vHip and $\mathrm{mPFC}$ is critical for both LTP and memory formation [22-24]. However, there is less evidence of the influence of over-activation of D1R in the vHip-mPFC circuity on learning and memory. A recent study suggested repeated stimulation of D1R in the dentate gyrus (DG) resulted in hippocampal plasticity deficits and hippocampusdependent long-term memory impairments [25]. As the inverted-
$\mathrm{U}$ action of prefrontal D1R affects working memory and short-term memory [26-28], it is not surprising to see the memory deficits in the chronic morphine-treated mice, which exhibit hyperactive D1R function.

We found that chronic morphine exposure increased $p$-ERK and p-CREB expression in the vHip and mPFC following D1R activation. These results are consistent with a previous study [29]. Our previous study has revealed that these protein levels in Hip are positively correlated with D1R activation during memory processes [24]. Although activation of D1R-ERK-CREB signaling in the striatum is critical for drug seeking behaviors, our data suggest D1R-ERK-CREB signaling within vHip-mPFC did not affect the morphine reward effect but only the cue/context-related responses. A recent study has revealed that both the vHip and mPFC project to the nucleus accumbens (NAC) shell D1R mediumsized spiny neurons (D1R-MSNs) but exert contrasting forms of synaptic plasticity during withdrawal from cocaine reward seeking 
[30]. These results suggest that the vHip and mPFC are involved in both context- and reward-related effects of abused drug but may recruit different populations and neural circuits.

One of our major findings in the present study is that the morphine reward memory switches to depend on D2R-Akt-GSK3 $\beta$ signaling within the vHip-mPFC circuit after chronic morphine experience. Although they receive less focus, D2R are also supposed to have a role in synaptic plasticity and Hipdependent learning and memory [31-33]. Reduced levels of pAkt and PGSK3 are observed in Hip and PFC after cocaine reward memory reactivation [34]. Our data suggest that integrated Akt-GSK3 $\beta$ signaling in the vHip-mPFC connection is required for morphine reward memory following chronic morphine exposure and withdrawal. Furthermore, blockage of $D 2 R$ in vHip-mPFC could restore pAkt (Thr308)/pGSK3 $\beta$ levels, inhibit morphine reinstatement, and alleviate spatial memory deficits in these mice, which is consistent with a previous study [34].

In the present study, we found an increase of pERK1 but not $p$ ERK2 in the vHip and mPFC after morphine-CPP formation. Although previous studies have suggested that blockage of ERK1/ 2 prevents long-lasting behavioral changes induced by morphine, including CPP $[35,36]$, there is evidence that ERK1 knockout mice exhibit enhanced responses to morphine-CPP [37]. A possible explanation for this findings is that ERK2 is the isoform necessary for gating the morphine-induced plasticity as suggested by Girault JA et al. [38]. It will be essential in future studies to direct evaluate the role played by ERK2 on morphine-associated contextual memory.

One limitation of the present study is that LTP is triggered by electrical stimulating the preserved hippocampal fiber bundle in mPFC. Although this method have shown efficiency in studying the synaptic plasticity of vHip-mPFC connection [18-20], it probably activate many other inputs to the mPFC and the effect observed could be partially mediated by other glutamatergic inputs to the mPFC such as mediodorsal thalamus and amygdala. Optogenetic stimulating that exact point in a coronal slice would activate only the vHip-mPFC fibers, pending further investigations.

In summary, we present evidence that D1R and D2R signaling in vHip-mPFC projections has key roles in morphine-related contextual memory formation and retrieval, respectively. Both D1R and D2R over-activation caused by morphine-CPP lead to impairments of Hip-dependent spatial memory, which can be alleviated by inhibiting D1R or D2R, respectively, largely depending on the morphine exposure states. These results uncovered a novel mechanism that integrates current knowledge on how changes in DA signaling within the Hip-PFC circuit during morphine dependence impacts normal memory formation through an opiate exposure state-dependent, DA receptorspecific manner.

\section{FUNDING}

This work was supported by the National Science Foundation of China (81501636, 81401108), the Basic Science Research Programs of Shaanxi Province (2016JQ8003), and the Postdoctoral Science Foundation of China (2015M582673).

\section{ADDITIONAL INFORMATION}

The online version of this article (https://doi.org/10.1038/s41386-018-0068-y) contains supplementary material, which is available to authorized users.

Competing interests: The authors declare no competing interests.

Publisher's note: Springer Nature remains neutral with regard to jurisdictional claims in published maps and institutional affiliations.

\section{REFERENCES}

1. Hyman SE. Addiction: a disease of learning and memory. Am J Psychiatry. 2005;162:1414-22.

2. Lisman JE, Grace AA. The hippocampal-VTA loop: controlling the entry of information into long-term memory. Neuron. 2005;46:703-13.

3. Pirot S, Godbout R, Mantz J, Tassin JP, Glowinski J, Thierry AM. Inhibitory effects of ventral tegmental area stimulation on the activity of prefrontal cortical neurons: evidence for the involvement of both dopaminergic and GABAergic components. Neuroscience. 1992;49:857-65.

4. McNamara CG, Dupret D. Two sources of dopamine for the hippocampus. Trends Neurosci. 2017;40:383-4.

5. Kutlu MG, Gould TJ. Effects of drugs of abuse on hippocampal plasticity and hippocampus-dependent learning and memory: contributions to development and maintenance of addiction. Learn Mem. 2016;23:515-33.

6. Gurden H, Takita M, Jay TM. Essential role of D1 but not D2 receptors in the NMDA receptor-dependent long-term potentiation at hippocampal-prefrontal cortex synapses in vivo. J Neurosci. 2000;20:RC106.

7. Goto Y, Grace AA. Dopamine modulation of hippocampal-prefrontal cortical interaction drives memory-guided behavior. Cereb Cortex. 2008;18:1407-14.

8. Esmaeili MH, Kermani M, Parvishan A, Haghparast A. Role of D1/D2 dopamine receptors in the CA1 region of the rat hippocampus in the rewarding effects of morphine administered into the ventral tegmental area. Behav Brain Res. 2012;231:111-5.

9. Assar N, Mahmoudi D, Farhoudian A, Farhadi MH, Fatahi Z, Haghparast A. D1- and D2-like dopamine receptors in the CA1 region of the hippocampus are involved in the acquisition and reinstatement of morphine-induced conditioned place preference. Behav Brain Res. 2016;312:394-404.

10. Tritsch NX, Sabatini BL. Dopaminergic modulation of synaptic transmission in cortex and striatum. Neuron. 2012;76:33-50.

11. Xing B, Li YC, Gao WJ. Norepinephrine versus dopamine and their interaction in modulating synaptic function in the prefrontal cortex. Brain Res. 2016;1641:217-33.

12. Beaulieu JM, Sotnikova TD, Marion S, Lefkowitz RJ, Gainetdinov RR, Caron MG. An Akt/beta-arrestin 2/PP2A signaling complex mediates dopaminergic neurotransmission and behavior. Cell. 2005;122:261-73.

13. Greengard P, Allen PB, Nairn AC. Beyond the dopamine receptor: the DARPP-32/ protein phosphatase-1 cascade. Neuron. 1999;23:435-47.

14. Kirschmann EK, Mauna JC, Willis CM, Foster RL, Chipman AM, Thiels E. Appetitive cue-evoked ERK signaling in the nucleus accumbens requires NMDA and D1 dopamine receptor activation and regulates CREB phosphorylation. Learn Mem. 2014;21:606-15.

15. Moron JA, Gullapalli S, Taylor C, Gupta A, Gomes I, Devi LA. Modulation of opiaterelated signaling molecules in morphine-dependent conditioned behavior: conditioned place preference to morphine induces CREB phosphorylation. Neuropsychopharmacology. 2010;35:955-66.

16. Miller JS, Barr JL, Harper LJ, Poole RL, Gould TJ, Unterwald EM. The GSK3 signaling pathway is activated by cocaine and is critical for cocaine conditioned reward in mice. PLoS ONE. 2014;9:e88026.

17. Wang $Y$, Lai J, Cui $H$, Zhu $Y$, Zhao B, Wang W, et al. Inhibition of histone deacetylase in the basolateral amygdala facilitates morphine context-associated memory formation in rats. J Mol Neurosci 2015;55:269-278.

18. Banks PJ, Burroughs AC, Barker GR, Brown JT, Warburton EC, Bashir ZI. Disruption of hippocampal-prefrontal cortex activity by dopamine D2R-dependent LTD of NMDAR transmission. Proc Natl Acad Sci USA. 2015;112:11096-101.

19. Parent MA, Wang L, Su J, Netoff T, Yuan LL. Identification of the hippocampal input to medial prefrontal cortex in vitro. Cereb Cortex. 2010;20:393-403.

20. Wang L, Yuan LL. Activation of M2 muscarinic receptors leads to sustained suppression of hippocampal transmission in the medial prefrontal cortex. J Physiol. 2009;587:5139-47.

21. Beaulieu JM, Tirotta E, Sotnikova TD, Masri B, Salahpour A, Gainetdinov RR, et al. Regulation of Akt signaling by D2 and D3 dopamine receptors in vivo. J Neurosci. 2007;27:881-5.

22. Hansen N, Manahan-Vaughan D. Dopamine D1/D5 receptors mediate informational saliency that promotes persistent hippocampal long-term plasticity. Cereb Cortex. 2014;24:845-58.

23. Puig MV, Antzoulatos EG, Miller EK. Prefrontal dopamine in associative learning and memory. Neuroscience. 2014;282:217-29.

24. Xing B, Kong H, Meng X, Wei SG, Xu M, Li SB. Dopamine D1 but not D3 receptor is critical for spatial learning and related signaling in the hippocampus. Neuroscience. 2010;169:1511-9.

25. Gangarossa G, Ceolin L, Paucard A, Lerner-Natoli M, Perroy J, Fagni L, et al. Repeated stimulation of dopamine D1-like receptor and hyperactivation of mTOR signaling lead to generalized seizures, altered dentate gyrus plasticity, and memory deficits. Hippocampus. 2014;24:1466-81. 
26. Vijayraghavan S, Wang M, Birnbaum SG, Williams GV, Arnsten AF. Inverted-U dopamine D1 receptor actions on prefrontal neurons engaged in working memory. Nat Neurosci. 2007;10:376-84.

27. Williams GV, Goldman-Rakic PS. Modulation of memory fields by dopamine D1 receptors in prefrontal cortex. Nature. 1995;376:572-5.

28. Xing B, Guo J, Meng X, Wei SG, Li SB. The dopamine D1 but not D3 receptor plays a fundamental role in spatial working memory and BDNF expression in prefrontal cortex of mice. Behav Brain Res. 2012;235:36-41.

29. Li SX, Wang ZR, Li J, Peng ZG, Zhou W, Zhou M, et al. Inhibition of Period1 gene attenuates the morphine-induced ERK-CREB activation in frontal cortex, hippocampus, and striatum in mice. Am J Drug Alcohol Abus. 2008:34:673-82.

30. Pascoli V, Terrier J, Espallergues J, Valjent E, O'Connor EC, Luscher C. Contrasting forms of cocaine-evoked plasticity control components of relapse. Nature. 2014;509:459-64.

31. Manahan-Vaughan D, Kulla A. Regulation of depotentiation and long-term potentiation in the dentate gyrus of freely moving rats by dopamine D2-like receptors. Cereb Cortex. 2003;13:123-35.

32. Nyberg L, Karalija N, Salami A, Andersson M, Wahlin A, Kaboovand N, et al. Dopamine D2 receptor availability is linked to hippocampal-caudate functional connectivity and episodic memory. Proc Natl Acad Sci USA. 2016;113:7918-23.
33. Takahashi H, Kato M, Takano H, Arakawa R, Okumura M, Otsuka T, et al. Differential contributions of prefrontal and hippocampal dopamine $\mathrm{D}(1)$ and $\mathrm{D}(2)$ receptors in human cognitive functions. J Neurosci. 2008;28:12032-8.

34. Shi X, Miller JS, Harper LJ, Poole RL, Gould TJ, Unterwald EM. Reactivation of cocaine reward memory engages the Akt/GSK3/mTOR signaling pathway and can be disrupted by GSK3 inhibition. Psychopharmacology. 2014;231:3109-18.

35. Lin X, Wang Q, Ji J, Yu LC. Role of MEK-ERK pathway in morphine-induced conditioned place preference in ventral tegmental area of rats. J Neurosci Res. 2010;88:1595-604.

36. Valjent E, Corbille AG, Bertran-Gonzalez J, Herve D, Girault JA. Inhibition of ERK pathway or protein synthesis during reexposure to drugs of abuse erases previously learned place preference. Proc Natl Acad Sci USA. 2006;103:2932-7.

37. Mazzucchelli C, Vantaggiato C, Ciamei A, Fasano S, Pakhotin P, Krezel W, et al Knockout of ERK1 MAP kinase enhances synaptic plasticity in the striatum and facilitates striatal-mediated learning and memory. Neuron. 2002;34:807-20.

38. Girault JA, Valjent E, Caboche J, Herve D. ERK2: a logical AND gate critical for drug-induced plasticity? Curr Opin Pharmacol. 2007;7:77-85. 